\title{
Life after treatment with external radiation therapy and brachytherapy in patients diagnosed with base of tongue cancer
}

\section{'Alexandra Oskarsson, 'Sandra Heimonen, ${ }^{2}$ Emma Ohlsson-Nevo}

${ }^{1}$ Faculty of Medicine and Health, Örebro University, Department of Oncology, Örebro University Hospital, Örebro, Sweden.

${ }^{2}$ Faculty of Medicine and Health, Örebro University, University Health Care Research Center, Örebro, Sweden.

\section{Background}

Brachytherapy is a treatment option for base of tongue cancer.

This includes external radiation- and brachytherapy in form of pulsed dose rated radiation, and in most cases a neck dissection.

Brachytherapy is given every two hours during 24 hours a day via permanent catheters during 3.5 days. The aim of the study was to describe how people diagnosed with base of tongue cancer experience the time after combination therapy with external radiation therapy and brachytherapy.

\section{Brachytherapy, base of tongue cancer}

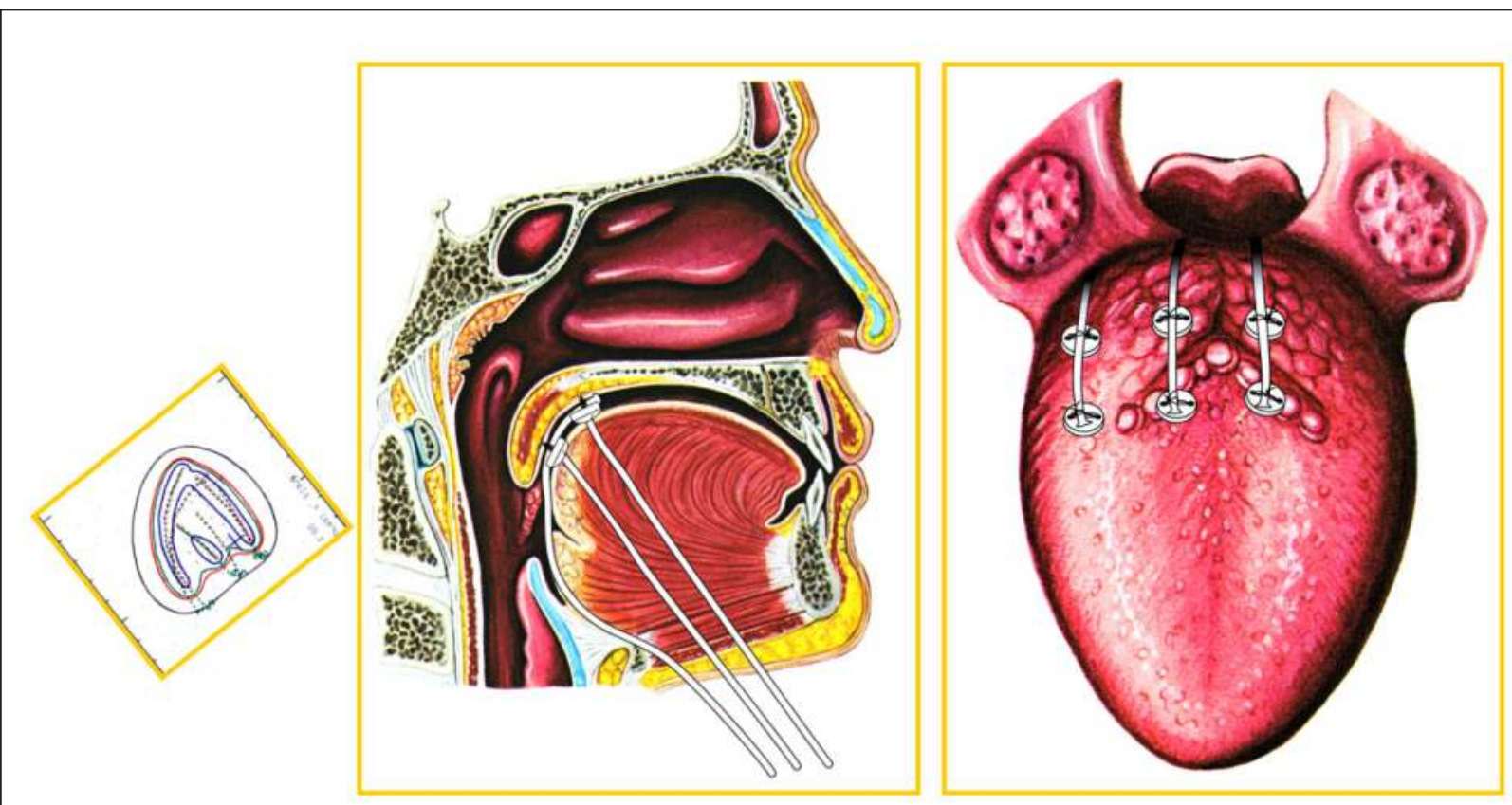

\section{Method}

The study has a qualitative method with a descriptive design. Data was collected by semi structured interviews. Eight participants who received brachytherapy against base of tongue cancer were interviewed.

The analysis was conducted with qualitative content analysis.

\section{Results}

The patients described how they had a changed body that led to a new life which required new solutions in their everyday life.

Dry mouth affected their life to a greater extent than they were expecting. The relationship to food and taste changed, as it lost its pleasure and changed their social context.

Their energy declined and the patients were unprepared for the increased inconvenience and the impact on life. Shoulder dysfunction after the neck dissection could limitate every day life.

Uncertainty about if the side effects would be permanent as well as an anxiety and fear that the cancer would return were present.

\section{Conclusion}

The results of this study highlights that the impact of the treatment is greater than the participants expected.

The result can provide insight into the problems that may arise for the patient after treatment as well as possibilities to tailor interventions targeting improvement in the information and care. 